\title{
Noise propagation in gene expression in the presence of decoys
}

\author{
Supravat Dey ${ }^{1}$, and Abhyudai Singh ${ }^{2}$
}

\begin{abstract}
Genetically-identical cells can show remarkable intercellular variability in the level of a given protein which is commonly known as the gene expression noise. Besides intrinsic fluctuations that arise from the inherent stochasticity of the biochemical processes, a significant source of expression noise is extrinsic. Such extrinsic noise in gene expression arises from cell-to-cell differences in expression machinery, transcription factors, cell size, and cell cycle stage. Here, we consider the synthesis of a transcription factor (TF) whose production is impacted by a dynamic extrinsic disturbance, and systematically investigate the regulation of expression noise by decoy sites that can sequester the protein. Our analysis shows that increasing decoy numbers reduce noise in the level of the free (unbound) TF with noise levels approaching the Poisson limit for large number of decoys. Interestingly, the suppression of expression noise compared to no-decoy levels is maximized at intermediate disturbance timescales. Finally, we quantify the noise propagation from the TF to a downstream target protein and find counterintuitive behaviors. More specifically, for nonlinear dose responses of target-protein activation, the noise in the target protein can increase with the inclusion of decoys, and this phenomenon is explained by smaller but more prolonged fluctuations in the TF level. In summary, our results illustrates the nontrivial effects of high-affinity decoys in shaping the stochastic dynamics of gene expression to alter cell fate and phenotype at the single-cell level.
\end{abstract}

\section{INTRODUCTION}

Genetically identical cells can show remarkable variability in the level of a gene product (mRNA/protein) which is commonly known as the gene expression noise [1]-[9]. Depending on the situation, the gene-expression noise can be detrimental or beneficial [10]-[14]. For example, large noise can cause defects in developing embryos [15]. Gene expression noise can drives different cell-fates of genetically identically cells [12]. Importantly, it can enhance phenotypic diversity, crucial for the survival of an organism in a population under fluctuating environments [13], [14].

There are two components of the cell-to-cell variability in gene expression - intrinsic and extrinsic [2], [3], [6], [7], [15]. The intrinsic noise arises from the inherent stochasticity of biochemical reactions associated with $\mathrm{mRNA}$ /protein productions and degradations involving few molecules. There are several cell-specific factors such as, cell cycle stage, cell size, abundance of expression machinery and global factors that are the extrinsic sources of expression noise [16][20]. The extrinsic and intrinsic noises can be quantified by experiments with two-color reporter genes that are regulated

1 Department of Electrical and Computer Engineering, University of Delaware, Newark, DE 19716, USA supravat.dey@gmail.com

${ }^{2}$ Department of Electrical and Computer Engineering, Department of Biomedical Engineering and Department of Mathematical Sciences, University of Delaware, Newark, DE 19716, USA absingh@udel. edu by identical promoters [2]. While correlated fluctuations represent the extrinsic noise, the uncorrelated fluctuations is a measure of intrinsic noise [2].

Here, we investigate the role of genomic decoy binding on the gene expression noise in the presence of a dynamic external disturbance. The genomic decoys are the numerous high-affinity binding sites where a transcription factors (TFs) bind without any direct functional consequences [21]. In functional binding, transcription factors bind to the specific site of a gene and directly regulate the expression by activating or inhibiting the transcription process. The binding of transcription factors to the decoy sites, on the other hand, alters the abundance of the transcription factor and indirectly regulates the expression of target genes. The role of decoys in the stochastic gene expression were studied experimentally using synthetic circuits in Saccharomyces cerevisiae and in various non-oscillatory circuits [22]-[27] and oscillatory circuits [28], [29] theoretically. However, the effect of decoys in the presence of external disturbance has not been addressed.

To investigate the role of decoys on the gene expression noise in the presence of a dynamic external perturbation, we formulate a simple stochastic model for the gene expression, schematically shown in Fig. 1. The transcription factor, whose production rate is subject to an external perturbation, reversibly binds to unoccupied decoy sites. Assuming small fluctuations in molecular copy numbers, we linearize associated binding terms around the means and solve the first and second-order moment dynamics at the steady-state. We quantify the noise in free TFs using the squared coefficient of variation and obtain analytical formulas for noise levels in the fast binding/unbinding limit. Our results show that the addition of decoy sites reduces the extrinsic noise component of the free TF level. Finally, using stochastic simulations, we investigate the noise for a downstream protein. Interestingly, we observe that decoys can enhance or buffer noise in the target protein depending on the nature of the dose response of the target protein activation.

Symbols and Notation: At a given time $t$, the number of molecules for the species associated with the external disturbance is denoted by $x(t)$, the molecular number of free and bound TFs by $y_{f}(t)$ and $y_{b}(t)$, and the number of target protein by $z(t)$. The molecular count of a species takes a random non-negative integer value i.e., $x(t), y_{f}(t), y_{b}(t), z(t) \in$ $\{0,1,2,3, \ldots\}$. For a stochastic process, we use angular brackets $\langle\cdot\rangle$ and $\overline{\langle\cdot\rangle}$ to denote the transient and steady state expected values respectively. The total noise in the molecular counts of a free TF is quantified by the square coefficient 
variation at the steady-state

$$
C V_{y}^{2}=\frac{\overline{\left\langle y_{f}\right\rangle}-{\overline{\left\langle y_{f}\right.}}^{2}}{{\overline{\left\langle y_{f}\right\rangle}}^{2}} .
$$

Similarly, we quantify noise in external disturbance and target protein count using $C V_{x}^{2}$ and $C V_{z}^{2}$, respectively.

\section{Modeling Gene EXPRESSION IN THE PRESENCE OF EXTRINSIC DISTURBANCES AND DECOY SITES}

We formulate a simple stochastic model schematically shown in Fig. 1, where the synthesis of TF is subject to an extrinsic disturbance. This disturbance biologically corresponds to fluctuations in the abundance of enzymes, expression machinery, or other global factors connected to cell size/cell-cycle stage. As discussed in further detail below, we phenomenologically model this disturbance as a bursty birth-death process.

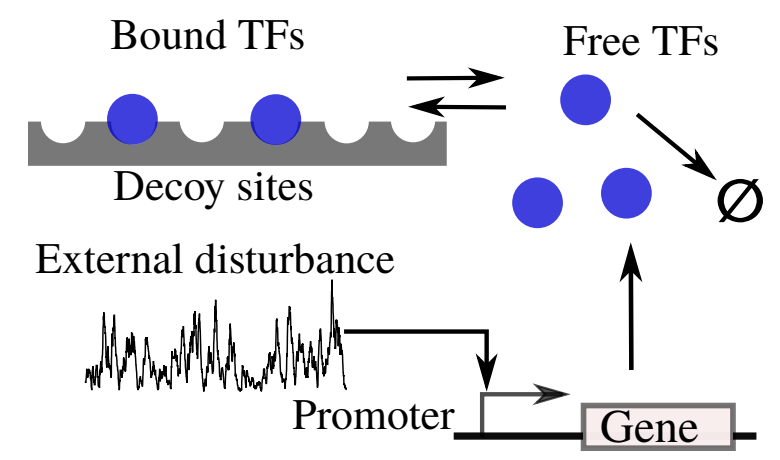

Fig. 1. Model schematic of TF expression impacted by upstream extrinsic noise. The synthesis of a transcription factor (TF) from a gene is modeled as a simple birthdeath process. An upstream disturbance affects the stochastic dynamics of TF copy numbers by making the synthesis rate itself a random process. There are $N$ decoy binding sites in the genome that can sequester the TF. A free TF molecule reversibly binds to an unoccupied decoy site with relatively fast binding/unbinding kinetics compared to the timescales of disturbance and $\mathrm{TF}$ turnover.

\section{A. Modeling extrinsic disturbance as a bursty process}

Increasing evidence shows that expression of gene products in single cells occurs in intermittent bursts [30]-[37], and bursty birth-death processes have been commonly used to capture fluctuations in the levels of these gene products [38]-[43]. Borrowing this framework, we model the extrinsic disturbance as a chemical species that is produces in bursts as per a Poisson process with rate $k_{x}$, and the species is subject to degradation at a constant rate. Let $x(t)$ denote the level of the disturbance at time $t$. Then, whenever a burst event occurs the level is reset by

$$
x \rightarrow x+B_{x}
$$

where the burst size $B_{x}$ is an independent and identically distributed random variable with $\operatorname{Prob}\left(B_{x}=i\right)=\alpha(i), i \in$
$\{1,2, \ldots\}$. Similarly, when a degradation event occurs the levels are reset as

$$
x \rightarrow x-1
$$

The probabilities of these rests occurring in the next infinitesimal time interval $(t, t+d t)$ are

$$
\begin{aligned}
& \operatorname{Prob}(x \rightarrow x+i)=k_{x} \alpha(i) d t \\
& \operatorname{Prob}(x \rightarrow x-1)=\gamma_{x} x d t,
\end{aligned}
$$

respectively, where $\gamma_{x}$ is the decay rate. For this system the mean and noise in molecular copy number $x(t)$ at steady state is given by

$$
\overline{\langle x\rangle}=\frac{k_{x}\left\langle B_{x}\right\rangle}{\gamma_{x}} \text { and } C V_{x}^{2}=\frac{\left\langle B_{x}\right\rangle+\left\langle B_{x}^{2}\right\rangle}{2\left\langle B_{x}\right\rangle \overline{\langle x\rangle}}
$$

respectively, where $\left\langle B_{x}\right\rangle$ is the average burst size [44], [45]. If $\left\langle B_{x}\right\rangle$ follows a geometric distribution with mean $\left\langle B_{x}\right\rangle$, then the second equation in (5) reduces to

$$
C V_{x}^{2}=\frac{\left\langle B_{x}\right\rangle}{\overline{\langle x\rangle}}
$$

and the magnitude of disturbance fluctuations increases with increasing $\left\langle B_{x}\right\rangle$. The speed of the fluctuations is given by the auto-correlation function

$$
R_{x}(\tau)=\frac{\langle x(\tau) x(0)\rangle-\overline{\left\langle x^{2}\right\rangle}}{\overline{\left\langle x^{2}\right\rangle}-\overline{\langle x\rangle}^{2}}=\mathrm{e}^{-\gamma_{x} \tau}
$$

[43], [44]. Thus, the degradation rate $\gamma_{x}$ is a measure of the speed or timescale of disturbance fluctuations. In other words, the fluctuations become fast as $\gamma_{x} \rightarrow \infty$, and become slow as $\gamma_{x} \rightarrow 0$. Next, we describe how this disturbance impacts the synthesis of a TF.

\section{B. Effect of extrinsic noise in the absence of decoy}

First, we discuss the effect of extrinsic noise on the stochastic synthesis of the TF. The dynamics of TF is assumed to follow a simple birth-death process with production rate that linearly depends on the disturbance, and a constant decay rate $\gamma_{y}$. Analogous to (4), but assuming no bursting, the probability of resets in the levels of the TF occurring in the next infinitesimal time interval $(t, t+d t)$ are

$$
\begin{aligned}
& \operatorname{Prob}\left(y_{f} \rightarrow y_{f}+1\right)=k_{y} \frac{x}{\langle x\rangle} d t \\
& \operatorname{Prob}\left(y_{f} \rightarrow y_{f}-1\right)=\gamma_{y} y_{f} d t,
\end{aligned}
$$

where $y_{f}(t)$ denotes the level of the TF at time $t$. To analyze the coupled random processes (8) and (4) we use the framework of moment dynamics for discrete-state continuous-time Markov processes as described in [46]. More specifically, the time evolution of any arbitrary function $\phi\left(x, y_{f}\right)=x^{m_{1}} y_{f}^{m_{2}}$ 


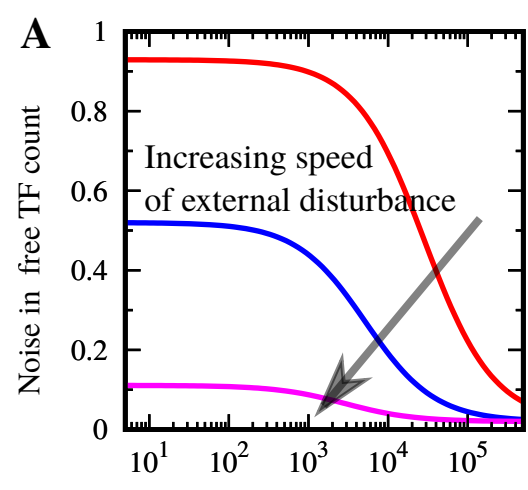

Number of decoys $(\mathrm{N})$

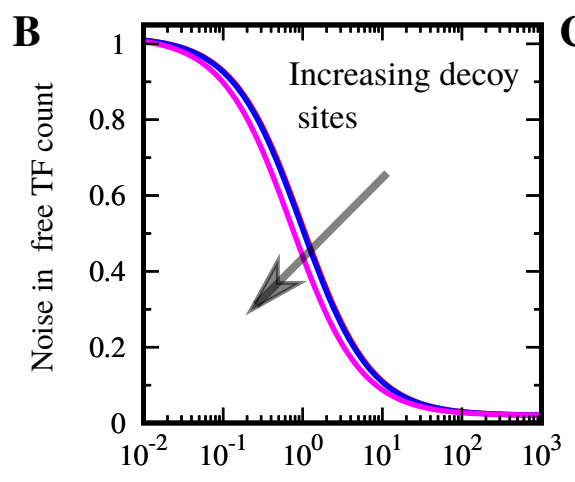

Speed of external disturbance $\left(\gamma_{\mathrm{X}}\right)$

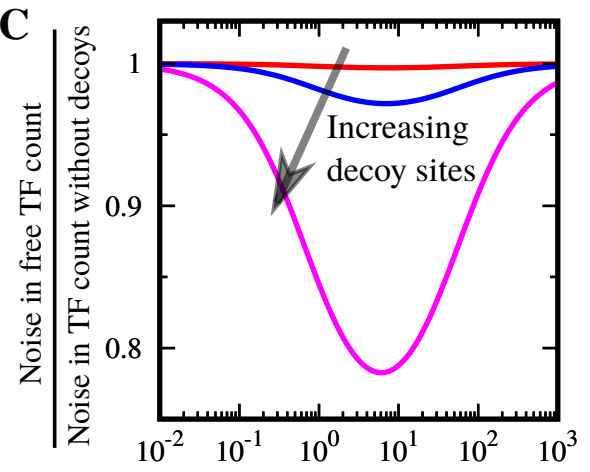

Speed of external disturbance $\left(\gamma_{\mathrm{X}}\right)$

Fig. 2. Decoy binding buffers gene expression noise due to extrinsic disturbance. (A) The noise in the free TF counts is plotted against $N$ for different values of speed of the external disturbance $\left(\gamma_{x}=0.1,1\right.$, and 10). The noise at $N=0$ is higher for a slower external disturbance. For large $N$, the noise approaches the Poisson limit. (B) The noise in the free TF counts is plotted against $\gamma_{x}$ for $N=10,100$, and 1000. The noise approaches to the Poisson limit for $\gamma_{x}>>\gamma_{y}$. (C) An optimal noise behavior is observed when the noise normalized by the noise level when $N=0$. The noise buffering becomes minimum for a particular value of $\gamma_{x}$. This minimum value of the noise decreases with increasing $N$. Parameter used: $\left\langle B_{x}\right\rangle=50, \overline{\langle x\rangle}=50, \overline{\left\langle y_{f}\right\rangle}=50, \gamma_{y}=1$, and $k_{d}=1$.

for $m_{1}, m_{2} \in\{0,1,2, \ldots\}$ is given by ,

$$
\begin{aligned}
\frac{d\left\langle\phi\left(x, y_{f}\right)\right\rangle}{d t} & =\left\langle\gamma_{x} x\left[\phi\left(x-1, y_{f}\right)-\phi\left(x, y_{f}\right)\right]\right\rangle \\
& +\left\langle\gamma_{y} y_{f}\left[\phi\left(x, y_{f}-1\right)-\phi\left(x, y_{f}\right)\right]\right\rangle \\
& +\left\langle k_{x} \sum_{i=0}^{\infty}\left[\phi\left(x+i, y_{f}\right)-\phi\left(x, y_{f}\right)\right]\right\rangle \\
& +\left\langle k_{y} \frac{x}{\langle x\rangle}\left[\phi\left(x, y_{f}+1\right)-\phi\left(x, y_{f}\right)\right]\right\rangle .
\end{aligned}
$$

We use this above extended generator to obtain the time evolution of all the first and second-order moments of $x(t)$ and $y_{f}(t)$. Solving these moment dynamics at steady-state reveals the following noise in the level of the TF (as quantified by its coefficient of variation squared)

$$
\overline{\left\langle y_{f}\right\rangle}=\frac{k_{y}}{\gamma_{y}} \text {, and } C V_{y}^{2}=\overbrace{\frac{1}{\overline{\left\langle y_{f}\right\rangle}}}^{\text {Intrinsic }}+\overbrace{C V_{x}^{2} \frac{\gamma_{y}}{\gamma_{x}+\gamma_{y}}}^{\text {Extrinsic }} .
$$

The first part of $C V_{y}^{2}$ is the intrinsic noise due to the Poissonian birth-death process, and the second part is the additional contribution due to the extrinsic noise. For a very fast external perturbation, $\gamma_{x} \rightarrow \infty$, the noise is purely intrinsic. For a very slow disturbance $\gamma_{x} \rightarrow 0, C V_{y}^{2}=$ $1 / \overline{\left\langle y_{f}\right\rangle}+C V_{x}^{2}$.

\section{Effect of extrinsic noise in the presence of decoy}

Next, we expand the model to include $N$ decoy binding sites. The free TF reversibly binds to unoccupied decoy sites with binding and unbinding rates $k_{b}$ and $k_{u}$, respectively. While a free TF molecule degrades at constant rate $\gamma_{y}$, we assume that TFs bound to decoys are protected from degradation [23], [25], [27].

Let random processes $y_{f}(t)$ and $y_{b}(t)$ denote the level of free and bound TF. Then, the probability of resets in $y_{f}(t)$ and $y_{b}(t)$ corresponding to different events occurring in $(t, t+d t)$ are as follows

$$
\begin{aligned}
& \operatorname{Prob}\left(y_{f} \rightarrow y_{f}+1\right)=k_{y} \frac{x}{\overline{\langle x\rangle}} d t \\
& \operatorname{Prob}\left(y_{f} \rightarrow y_{f}-1\right)=\gamma_{y} y_{f} d t \\
& \operatorname{Prob}\left(y_{f} \rightarrow y_{f}-1 ; y_{b} \rightarrow y_{b}+1\right)=k_{b}\left(N-y_{b}\right) y_{f} d t \\
& \operatorname{Prob}\left(y_{f} \rightarrow y_{f}+1 ; y_{b} \rightarrow y_{b}-1\right)=k_{u} d t .
\end{aligned}
$$

Here the first two resets represent the synthesis and degradation of a free TF molecule, and the last two resets represent binding/unbinding to decoys. Note that $N-y_{b}$ is the number of unoccupied decoys resulting in the nonlinear binding rate is $k_{b}\left(N-y_{b}\right) y_{f}$. Combing (11) with (4) yields the following extended generator for any arbitrary function $\phi\left(x_{f}, y_{f}, y_{b}\right)$ :

$$
\begin{aligned}
& \frac{d\left\langle\phi\left(x, y_{f}, y_{b}\right)\right\rangle}{d t}=\left\langle\gamma_{x} x\left[\phi\left(x-1, y_{f}, y_{b}\right)-\phi\left(x, y_{f}, y_{b}\right)\right]\right\rangle \\
& \quad+\left\langle\gamma_{y} y\left[\phi\left(x, y_{f}-1, y_{b}\right)-\phi\left(x, y_{f}, y_{b}\right)\right]\right\rangle \\
& +\left\langle k_{x} \sum_{i=0}^{\infty}\left[\phi\left(x+i, y_{f}, y_{b}\right)-\phi\left(x, y_{f}, y_{b}\right)\right]\right\rangle \\
& \quad+\left\langle k_{y} \frac{x}{\langle x\rangle}\left[\phi\left(x, y_{f}+1, y_{b}\right)-\phi\left(x, y_{f}, y_{b}\right)\right]\right\rangle \\
& +\left\langle k_{b} y_{f}\left(N-y_{b}\right)\left[\phi\left(x, y_{f}-1, y_{b}+1\right)-\phi\left(x, y_{f}, y_{b}\right)\right]\right\rangle \\
& +\left\langle k_{u} y_{b}\left[\phi\left(x, y_{f}+1, y_{b}-1\right)-\phi\left(x, y_{f}, y_{b}\right)\right]\right\rangle,
\end{aligned}
$$

and time evolution of moments is obtained by setting $\phi\left(x_{f}, y_{f}, y_{b}\right)=x^{m_{1}} y_{f}^{m_{2}} y_{b}^{m_{3}}$ for $m_{1}, m_{2}, m_{3} \in\{0,1,2, \ldots\}$ [46]. It turns out that the nonlinear binding rate results in the problem of unclosed dynamics - time evolution of lower order moments depends on higher order moments, and typically moment closure schemes are exploited to compute moments. Here we use the well-known linear noise approximation [48]-[51] that under the assumption of small fluctuations in $y_{f}$ and $y_{b}$ around their respective mean values $\left\langle y_{f}\right\rangle$ and $\left\langle y_{b}\right\rangle$, linearizes the nonlinearity as

$$
k_{b} y_{b} y_{f} \approx k_{b}\left(y_{b}\left\langle y_{f}\right\rangle+y_{f}\left\langle y_{f}\right\rangle-\left\langle y_{b}\right\rangle\left\langle y_{f}\right\rangle\right) .
$$



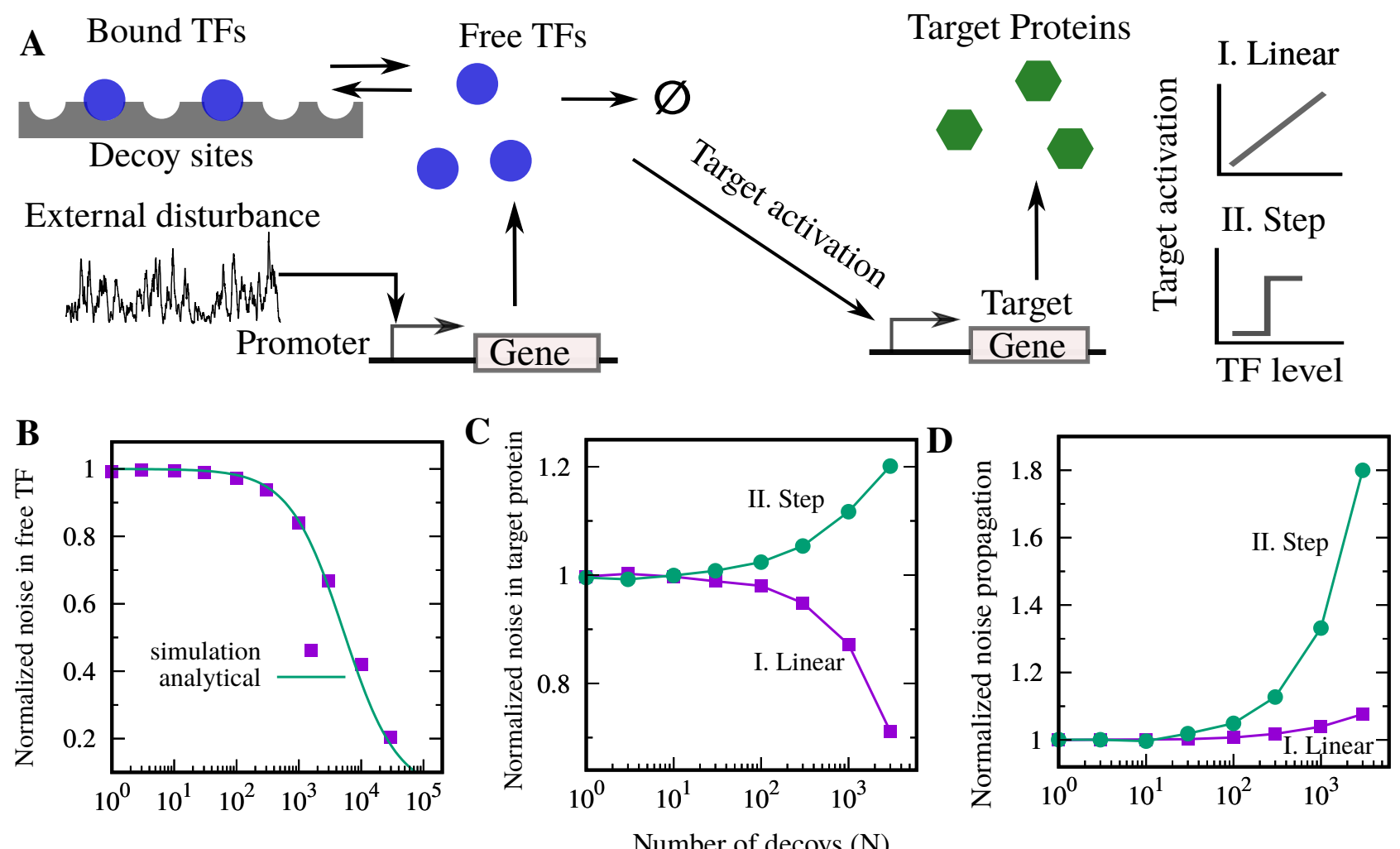

Fig. 3. Target protein noise from stochastic simulations shows a distinct behavior depending on linear and step-like regulation. (A) A schematic diagram of the model for studying noise in the downstream gene expression. Free TFs activate the expression of the target gene: production rate is $k_{z} g\left(y_{f}\right)$. We consider two cases: (I) Linear target activation: $g\left(y_{f}\right)=y_{f}$, and (II) Step-function target activation: $g\left(y_{f}\right)=\Theta\left(y_{f}-\overline{\left\langle y_{f}\right\rangle}\right)$, where $\Theta$ is the Heaviside step-function, i.e., $\Theta(x)=0$ when $x<0$ and $\Theta(x)=1$ when $x>0$. (B) The results of the noise in free TF counts obtained from running a large number of Monte Carlo simulations using the Stochastic Simulation Algorithm [47] and analytical formula (15) are in agreement. (C) The noise in the target protein (normalized by $C V_{z}^{2}(N=0)$ ) plotted against $N$. While for the linear regulation, the noise in the target protein decreases, for the step-like regulation noise in the target protein increases as a function of $N$. (D) Noise propagation defined as the ratio of free TF noise level to the target protein noise level increases as function of $N$. However, the noise propagation is higher for the step-like dose response. For this plot parameters taken as $\left\langle B_{x}\right\rangle=50$, $k_{x}=1, k_{y}=50, \gamma_{x}=\gamma_{y}=\gamma_{z}=1, k_{d}=1$, and $k_{z}=1$.

Using these linearized rates in place of the nonlinearity $k_{b} y_{b} y_{f}$ in (12), one can again derive the time evolution of the moments and solve them to get approximate analytical formulas for the TF noise levels. Given the space constraints, we omit the proof and only present the main results.

Solving the first order moment dynamics at the steadystate, we obtain the expressions for the mean free TF and bound TF counts,

$$
\overline{\left\langle y_{f}\right\rangle}=\frac{k_{y}}{\gamma_{y}} \text { and } \overline{\left\langle y_{b}\right\rangle}=\frac{N \overline{\left\langle y_{f}\right\rangle}}{\overline{\left\langle y_{f}\right\rangle}+k_{d}}
$$

where, $k_{d}=k_{u} / k_{b}$ is the dissociation constant. Please note that $\overline{\left\langle y_{f}\right\rangle}$ is independent of the decoy sites as has been shown before in the absence of disturbance [25]. Solving the second order moment dynamics, we obtain the following formula for the noise in the free TF in the limit of fast binding/unbinding $\left(k_{b} \rightarrow \infty, k_{u} \rightarrow \infty\right.$ and $k_{d}=k_{u} / k_{b}$ being finite),

$C V_{y}^{2}=\overbrace{\frac{1}{\overline{\left\langle y_{f}\right\rangle}}}^{\text {Intrinsic }}+\overbrace{C V_{x}^{2} \frac{\overline{\left\langle y_{f}\right\rangle} \gamma_{y}}{N f(1-f) \gamma_{x}+\overline{\left\langle y_{f}\right\rangle}\left(\gamma_{x}+\gamma_{y}\right)}}^{\text {Extrinsic }}$,

where $f=\overline{\left\langle y_{b}\right\rangle} / N$ is the fraction of bound TFs. Note that decoy binding directly affect the extrinsic noise part. The noise decreases monotonically to the Poisson limit $1 / \overline{\left\langle y_{f}\right\rangle}$ as $N \rightarrow \infty$. Fig. 2(A) shows how the noise decays with $N$ for various values of the speed of the extrinsic (disturbance) fluctuations $\gamma_{x}$. For a given $N, C V_{y}^{2}=1 / \overline{\left\langle y_{f}\right\rangle}+C V_{x}^{2}$ for $\gamma_{x}<<\gamma_{y}$ and $C V_{y}^{2}=1 / \overline{\left\langle y_{f}\right\rangle}$ for $\gamma_{x}<<\gamma_{y}$ (see Fig. 2(B)). If we plot the noise in free TF normalized with that of $N=0$ as a function of $\gamma_{x}$, the noise show a minima for a specific value of $\gamma_{x}$ (see Fig. 2(C)). In essence, the noise suppression ability of decoys is highest at intermediate timescales of disturbance fluctuations. 


\section{NOISE IN THE TARGET PROTEIN}

Having quantified the effect of decoys on TF expression noise, we next study noise propagation from the TF to a downstream target gene. Towards that end, free TF molecules activate the production of a target protein with synthesis rate $k_{z} g\left(y_{f}\right)$ (Fig. 3(A)). We study two specific cases: (I) Linear activation, $g\left(y_{f}\right)=k_{z} y_{f}$ and (II) Step-like activation (activation only occurs when $\left.y_{f}>\overline{\left\langle y_{f}\right\rangle}\right), g\left(y_{f}\right)=\Theta\left(y_{f}-\right.$ $\left.\overline{\left\langle y_{f}\right\rangle}\right)$, where $\Theta$ is the Heaviside step-function, i.e., $\Theta(x)=0$ when $x<0$ and $\Theta(x)=1$ when $x>0$. As in the case of the $\mathrm{TF}$, assuming a non-bursty production of the target protein results in the following probability of resets

$$
\begin{aligned}
& \operatorname{Prob}(z \rightarrow z+1)=k_{z} g\left(y_{f}\right) d t \\
& \operatorname{Prob}(z \rightarrow z-1)=\gamma_{z} z d t .
\end{aligned}
$$

for the birth and death of the target protein, where $z(t)$ denotes the level of the target protein, and $\gamma_{z}$ is the target protein's degradation rate. An important point to mention is that only the TF binds to decoys. A key focus here is to quantify the extent of noise propagation from the TF to the target protein as a function of decoy numbers for different dose responses $g\left(y_{f}\right)$.

We study this system of four nonlinearly coupled random processes $x(t), y_{f}(t), y_{b}(t)$ and $z(t)$ by running a large number of Monte Carlo simulations using the Stochastic Simulation Algorithm [47]. Quantification of steady-state level noise levels for different species are shown Fig. 3. First, we show that the analytical result of noise in the free TF (15) as obtained using the linear noise approximation matches nicely with the simulations (Fig. 3(B)). The noise in the target protein $C V_{z}^{2}$ shows a counterintuitive behavior. Given that decoys buffers noise in free TF, one might expect the same behavior in the target protein noise. However, we see two opposite role of decoys depending on the profiles of the activation. For a linear dose response, decoys buffer noise in the target protein, but enhance noise for the step-like dose response (Fig. 3).

How does the net noise propagation to the target protein behave? The net propagation of noise from TF to target protein can be measured by $C V_{z}^{2} / C V_{y}^{2}$. It is interesting to see that although $C V_{z}^{2}$ shows a distinct behavior, the propagation of noise shows enhancements for both the cases (Fig. 3(D)). However, it should be noted that noise propagation is significantly higher for the step-like dose response.

To better understand these results we decided to focus on the timescale of fluctuations of the free TF level in the presence of decoys. We computed the auto-correlation function of the free TF level using stochastic trajectories at steady-state as per

$$
R_{y}(\tau)=\frac{\left\langle y_{f}(\tau) y_{f}(0)\right\rangle-{\overline{\left\langle y_{f}\right.}}^{2}}{\overline{\left\langle y_{f}^{2}\right\rangle}-{\overline{\left\langle y_{f}\right\rangle}}^{2}},
$$

A slower decay of $R_{y}(\tau)$ enhances noise propagation but a faster decay reduces noise propagation [25], [52]. In Fig. 4, we plot auto-correlation function for $N=0,1000$, and
3000 revealing that the decay of $R_{y}$ becomes slower for larger decoy abundances, explaining the enhancement in the noise propagation seen in both the linear and step-like dose responses (Fig .3). Why is the noise propagation higher for the step-like dose response? Note that for a step-like dose response, noise propagation to the target protein only depends on the speed of fluctuations in $y_{f}(t)$, i.e., how fast TF levels go below the threshold and bounce back. In contrast, for a linear dose response, noise propagation depends both on the speed of fluctuations and the magnitude of fluctuations in the free TF level. As the fee TF noise levels decrease with increasing $N$ (Figs. 2 and 3), they buffer the noise propagation in the linear case, but not in the step-like nonlinear case.

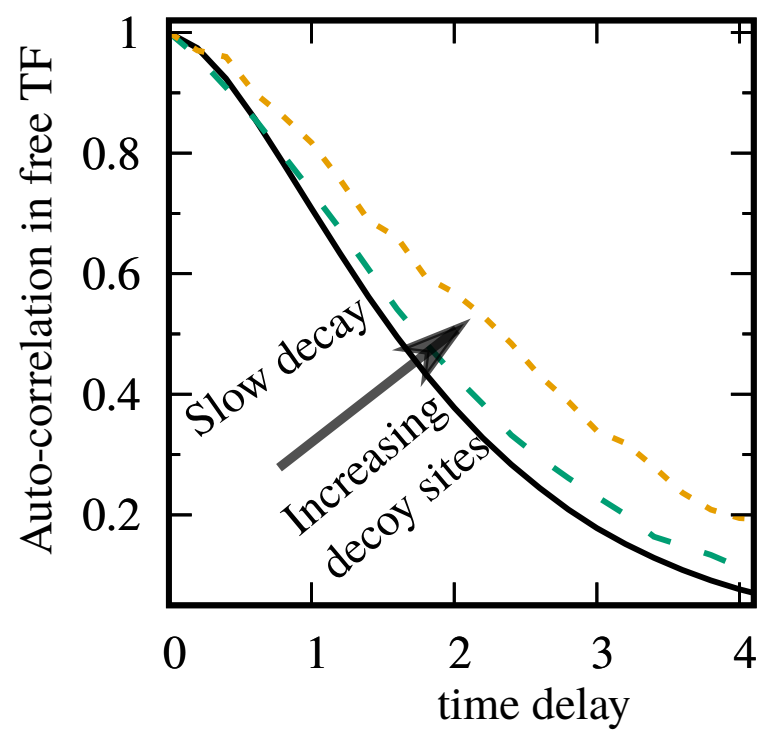

Fig. 4. Decoy slow the speed of the fluctuations in the levels of the free TF. Auto-correlation function $R_{y}$ for free $\mathrm{TF}$ as a function of time for $N=0,1000$, and 3000 . The decay of $R_{y}$ becomes slower for larger $N$. Parameter used: $\left\langle B_{x}\right\rangle=50, k_{x}=1, k_{y}=50, \gamma_{x}=\gamma_{y}=1$, and $k_{d}=1$.

\section{CONCLUSION}

A significant portion of gene expression noise is extrinsic and can propagate downstream to target proteins with important consequences for cellular functioning. We have investigated the role of decoy binding sites on the propagation of extrinsic noise to downstream proteins. For this, we have formulated a stochastic model where a dynamical extrinsic disturbance modulates the synthesis rate of a TF, and free TF molecules can bind to genomic decoy binding sites that are present in a fixed number.

We have obtained analytical formula of noise in free TF counts in the limit of fast binding/unbinding, assuming small fluctuations around the mean. We have observed that free TF noise levels decrease as a function of the total number of decoy sites, and approach the Poisson limit for large decoy abundances (Fig. 2). Interestingly, the noise suppression 
bioRxiv preprint doi: https://doi.org/10.1101/2020.04.01.020032; this version posted April 2, 2020. The copyright holder for this preprint (which was not certified by peer review) is the author/funder, who has granted bioRxiv a license to display the preprint in perpetuity. It is made available under aCC-BY-ND 4.0 International license.

ability of decoys is highest at intermediate timescales of disturbance fluctuations.

Finally, we have analyzed noise propagation to a target protein by stochastic simulations. Surprisingly, the noise in the target protein shows an enhancement with the addition of decoy sites when its activation by a TF occurs digitally beyond a threshold (Fig. 3). Thus, while increasing decoys decrease noise in the free TF level, it increases noise in the target protein. We argue that this increase in noise is explained by a slowing of fluctuations in the free TF level (Fig. 4), and hence the random switch between turning target proteins expression on and off occurs more slowly. A key focus of future work will be to develop theoretical tools to quantify noise propagation and systematically explore them for different dose-response shapes.

\section{ACKNOWLEDGMENT}

This work is supported by the National Science Foundation Grant ECCS-1711548.

\section{REFERENCES}

[1] H. H. McAdams and A. P. Arkin, "Stochastic mechanisms in gene expression," Proceedings of the National Academy of Sciences, vol. 94, pp. 814-819, 1997.

[2] M. B. Elowitz, A. J. Levine, E. D. Siggia, and P. S. Swain, "Stochastic gene expression in a single cell," Science, vol. 297, pp. 1183-1186, 2002.

[3] P. S. Swain, M. B. Elowitz, and E. D. Siggia, "Intrinsic and extrinsic contributions to stochasticity in gene expression," Proceedings of the National Academy of Sciences, vol. 99, pp. 12 795-12 800, 2002.

[4] E. M. Ozbudak, M. Thattai, I. Kurtser, A. D. Grossman, and A. van Oudenaarden, "Regulation of noise in the expression of a single gene," Nature Genetics, vol. 31, pp. 69-73, 2002.

[5] W. J. Blake, M. Kaern, C. R. Cantor, and J. J. Collins, "Noise in eukaryotic gene expression," Nature, vol. 422, pp. 633-637, 2003.

[6] M. Scott, B. Ingalls, and M. Kaern, "Estimations of intrinsic and extrinsic noise in models of nonlinear genetic networks," CHAOS, vol. 16, p. 026107, 2006.

[7] V. Shahrezaei, J. F. Ollivier, and P. S. Swain, "Colored extrinsic fluctuations and stochastic gene expression," Molecular Systems Biology, vol. 4, 2008.

[8] A. Eldar and M. B. Elowitz, "Functional roles for noise in genetic circuits," Nature, vol. 467, pp. 167-173, 2010.

[9] B. Munsky, G. Neuert, and A. van Oudenaarden, "Using gene expression noise to understand gene regulation," Science, vol. 336, pp. 183-187, 2012.

[10] J. M. Raser and E. K. O'Shea, "Noise in gene expression: Origins, consequences, and control," Science, vol. 309, pp. 2010 - 2013, 2005.

[11] A. Raj and A. van Oudenaarden, "Nature, nurture, or chance: stochastic gene expression and its consequences," Cell, vol. 135, pp. 216-226, 2008.

[12] R. Losick and C. Desplan, "Stochasticity and cell fate," Science, vol. 320, pp. 65-68, 2008.

[13] C. J. Davidson and M. G. Surette, "Individuality in bacteria," Annual Review of Genetics, vol. 42, pp. 253-268, 2008.

[14] D. Fraser and M. Kaern, "A chance at survival: gene expression noise and phenotypic diversification strategies," Mol Microbiol, vol. 71, pp. 1333-1340, 2009.

[15] S. Keskin, G. S. Devakanmalai, S. B. Kwon, H. Vu, M. Soltani, A. Singh, A. Ay, and E. M. Ozbudak, "Noise in the vertebrate segmentation clock is boosted by time-delays but tamed by notch signaling," Cell Reports, vol. 23, pp. 2175-2185, 2018.

[16] O. Padovan-Merhar, G. P. Nair, A. G. Biaesch, A. Mayer, S. Scarfone, S. W. Foley, A. R. Wu, L. S. Churchman, A. Singh, and A. Raj, "Single Mammalian Cells Compensate for Differences in Cellular Volume and DNA Copy Number through Independent Global Transcriptional Mechanisms," Molecular Cell, vol. 58, no. 2, pp. 339-352, 2015.
[17] L. Keren, D. Van Dijk, S. Weingarten-Gabbay, D. Davidi, G. Jona, A. Weinberger, R. Milo, and E. Segal, "Noise in gene expression is coupled to growth rate," Genome Research, vol. 25, no. 12, pp. 18931902, 2015.

[18] B. A. C. M. S. Sherman, K. Lorenz, M. Hunter Lanier, "Correlated Fluctuations in Gene Expression," Cell Syst., vol. 1, no. 5, pp. 315$325,2016$.

[19] A. Baudrimont, V. Jaquet, S. Wallerich, S. Voegeli, and A. Becskei, "Contribution of RNA Degradation to Intrinsic and Extrinsic Noise in Gene Expression," Cell Reports, vol. 26, no. 13, pp. 3752-3761.e5, 2019.

[20] X.-M. Sun, A. Bowman, M. Priestman, F. Bertaux, A. MartinezSegura, W. Tang, C. Whilding, D. Dormann, V. Shahrezaei, and S. Marguerat, "Size-Dependent Increase in RNA Polymerase II Initiation Rates Mediates Gene Expression Scaling with Cell Size," Current Biology, pp. 1-14, 2020. [Online]. Available: https://doi.org/10.1016/j.cub.2020.01.053

[21] C. A. Kemme, D. Nguyen, A. Chattopadhyay, and J. Iwahara, "Regulation of transcription factors via natural decoys in genomic dna," Transcription, vol. 7, no. 4, pp. 115-120, 2016.

[22] T. Lee and N. Maheshri, "A regulatory role for repeated decoy transcription factor binding sites in target gene expression," Molecular systems biology, vol. 8, p. 576, 2012.

[23] A. Burger, A. M. Walczak, and P. G. Wolynes, "Abduction and asylum in the lives of transcription factors," Proceedings of the National Academy of Sciences, vol. 107, pp. 4016-4021, 2010.

[24] — - "Influence of decoys on the noise and dynamics of gene expression," Phes. Rev. E, vol. 86, p. 041920, 2012.

[25] M. Soltani, P. Bokes, Z. Fox, and A. Singh, "Nonspecific transcription factor binding can reduce noise in the expression of downstream proteins," Physical Biology, vol. 12, p. 055002, 2015.

[26] D. Das, S. Dey, R. C. Brewster, and S. Choube, "Effect of transcription factor resource sharing on gene expression noise," PLoS Comput Biol, vol. 13, p. e1005491, 2017.

[27] S. Dey and A. Singh, "Stochastic analysis of feedback control by molecular sequestration," in 2019 American Control Conference (ACC), 2019, pp. 4466-4471.

[28] S. Jayanthi and D. Del Vecchio, "Tuning genetic clocks employing DNA binding sites," PLOS ONE, vol. 7, p. e41019, 2012.

[29] Z. Wang, D. A. Potoyan, and P. G. Wolynes, "Molecular stripping, targets and decoys as modulators of oscillations in the nf-kb/ikba/dna genetic network," J R Soc Interface, vol. 13, no. 122, p. 20160606, 2016.

[30] D. M. Suter, N. Molina, D. Gatfield, K. Schneider, U. Schibler, and F. Naef, "Mammalian genes are transcribed with widely different bursting kinetics," Science, vol. 332, pp. 472-474, 2011.

[31] R. D. Dar, B. S. Razooky, A. Singh, T. V. Trimeloni, J. M. McCollum, C. D. Cox, M. L. Simpson, and L. S. Weinberger, "Transcriptional burst frequency and burst size are equally modulated across the human genome," Proceedings of the National Academy of Sciences, vol. 109, pp. 17454-17459, 2012.

[32] T. Fukaya, B. Lim, and M. Levine, "Enhancer control of transcriptional bursting," Cell, vol. 166, pp. 358-368, 2015.

[33] C. R. Bartman, S. C. Hsu, C. C.-S. Hsiung, A. Raj, and G. A. Blobel, "Enhancer regulation of transcriptional bursting parameters revealed by forced chromatin looping," Molecular Cell, vol. 62, pp. 237 - 247, 2016.

[34] A. M. Corrigan, E. Tunnacliffe, D. Cannon, and J. R. Chubb, "A continuum model of transcriptional bursting," eLife, vol. 5, p. e13051, 2016.

[35] S. Chong, C. Chen, H. Ge, and X. S. Xie, "Mechanism of transcriptional bursting in bacteria," Cell, vol. 158, pp. 314-326, 2014.

[36] A. Singh, B. Razooky, C. D. Cox, M. L. Simpson, and L. S. Weinberger, "Transcriptional bursting from the HIV-1 promoter is a significant source of stochastic noise in HIV-1 gene expression," Biophysical Journal, vol. 98, pp. L32-L34, 2010.

[37] R. D. Dar, S. M. Shaffer, A. Singh, B. S. Razooky, M. L. Simpson, A. Raj, and L. S. Weinberger, "Transcriptional bursting explains the noise-versus-mean relationship in mRNA and protein levels," PLOS ONE, vol. 11, p. e0158298, 2016.

[38] N. Friedman, L. Cai, and X. Xie, "Linking stochastic dynamics to population distribution: an analytical framework of gene expression," Physical Review Letters, vol. 97, p. 168302, 2006.

[39] V. Shahrezaei and P. S. Swain, "Analytical distributions for stochastic 
gene expression," Proceedings of the National Academy of Sciences, vol. 105, pp. 17256-17261, 2008.

[40] N. Kumar, T. Platini, and R. V. Kulkarni, "Exact Distributions for Stochastic Gene Expression Models with Bursting and Feedback," Physical Review Letters, vol. 113, p. 268105, 2014.

[41] A. Singh and P. Bokes, "Consequences of mRNA transport on stochastic variability in protein levels," Biophysical Journal, vol. 103, pp. 1087-1096, 2012.

[42] N. Kumar, A. Singh, and R. V. Kulkarni, "Transcriptional bursting in gene expression: Analytical results for genera stochastic models," PLOS Computational Biology, vol. 11, p. e1004292, 2015.

[43] J. M. Pedraza and J. Paulsson, "Effects of molecular memory and bursting on fluctuations in gene expression," Science, vol. 319, pp. $339-343,2008$.

[44] A. Singh and M. Soltani, "Quantifying intrinsic and extrinsic variability in stochastic gene expression models," PLOS ONE, vol. 8, p. e84301, 2013.

[45] S. Modi, S. Dey, and A. Singh, "Proportional and derivative controllers for buffering noisy gene expression," in 2019 IEEE 58th Conference on Decision and Control (CDC), 2019, pp. 2832-2837.
[46] A. Singh and J. P. Hespanha, "Approximate moment dynamics for chemically reacting systems," IEEE Transactions on Automatic Control, vol. 56, pp. 414-418, 2011.

[47] D. T. Gillespie, "Exact stochastic simulation of coupled chemical reactions," Journal of Physical Chemistry, vol. 81, pp. 2340-2361, 1977.

[48] N. Van Kampen, Stochastic Processes in Physics and Chemistry. Elsevier, 2011.

[49] S. Modi, M. Soltani, and A. Singh, "Linear noise approximation for a class of piecewise deterministic markov processes," in 2018 Annual American Control Conference (ACC), 2018, pp. 1993-1998.

[50] J. Elf and M. Ehrenberg, "Fast evaluation of fluctuations in biochemical networks with the linear noise approximation," Genome Research, vol. 13, pp. 2475-2484, 2003.

[51] P. Thomas, A. V. Straube, and R. Grima, "The slow-scale linear noise approximation: an accurate, reduced stochastic description of biochemical networks under timescale separation conditions," $B M C$ Systems Biology, vol. 6, p. 39, 2012.

[52] S. Dey, M. Soltani, and A. Singh, "Enhancement of gene expression noise due to nonspecific transcription factor binding," bioRxiv, 2019. 